\title{
TEM Studies of Au/Si Epilayer Interfaces
}

Feng Wang, 'Sayed Y. Sayed,, ** Marek Malac, , *** Dong Wang, Ray Egerton,, *** and Jillian Buriak $^{*}, * *$

* National Institute for Nanotechnology, Edmonton, T6G 2M9, Canada

** Department of Chemistry, University of Alberta, Edmonton, T6G 2G2, Canada

*** Department of Physics, University of Alberta, Edmonton, T6G 2G7, Canada

There is much interest in fabricating metallic contacts on semiconductor surfaces by a straightforward class of electrochemical reactions called galvanic displacement [1, 2]. The chemistry is carried out via contact of the desired semiconductor with the metal ion solution (generally aqueous); the semiconductor acts as the source of electrons, reducing the metal ions in situ to metal. In the case of gold deposition on silicon, we have evidence that the growth of $\mathrm{Au}$ nanoparticles on silicon is epitaxial [3]. In order to better understand the nature of these interfaces, we carried out a series of plan-view and cross-section transmission electron microscopy (TEM) studies. TEM samples were prepared by mechanical grinding followed with ion milling at cryogenic temperatures (below $-100^{\circ} \mathrm{C}$ ). TEM images and diffraction patterns were recorded using a 200kV JEOL 2200FS TEM/STEM instrument.

Figure 1 shows the morphology of the $\mathrm{Au}$ nanoparticles/islands on $\mathrm{Si}(111)$ surfaces in a secondary electron image; periodic anodic (black) and cathodic (grey) sites are well resolved and can be attributed to local nature of the redox process. The epitaxial growth of the Au nanoparticle islands are well demonstrated by the moiré fringes in plan view TEM images and the corresponding selected area electron diffraction (SAED) taken near the [112] direction in Fig. 2. Similar to the case of Ag nanoparticles vapor-deposited on Si, the dense-packed Au\{111\} planes tend to align with $\operatorname{Si}(111)$ [4]. So-called domain-matching epitaxy appears to accommodate the large lattice mismatch between $\mathrm{Au}$ and $\mathrm{Si}$ [5]. Details of the atomic arrangement are provided by cross-section TEM images as in Fig. 3. V-shaped Au particles are formed, with every four $\mathrm{Au}$ atomic planes matched with three Si planes (dotted-lines in the figure provide a guidance of the lattice matching).

In order to complement the work described above on flat silicon, we have also investigated $\mathrm{Au}$ nanoparticles deposited on Si nanowires. Au particles have a strong tendency to grow on certain facets, such as (110) for particles on a <112> nanowire; this is demonstrated in Fig. 4. By using a nano beam probe, TEM images and electron diffraction patterns were acquired from the interface area, allowing us to measure relative orientation of $\mathrm{Si}$ and $\mathrm{Au}$ lattices. While straightforward to carry out, the underlying mechanism of galvanic deposition is complicated and thus the reason for the selectivity is not yet known at this time [2]; nevertheless, the preferential growth may be dictated by the interfacial interaction of the $\mathrm{Au} / \mathrm{Si}$ epilayers. More control experiments and theoretical calculations are under way to further investigate this issue [6].

\section{References}

[1] L.A. Porter, C.H. Chiou, A.E. Ribbe, and J.M. Buriak, Nano. Lett. 2, 1067, (2002).

[2] G. Oskam, J.G. Long, A. Natarajan, P.C. Searson, J. Phys. D: Appl.Phys. 31, 1927 (1998).

[3] S.Y. Sayed., F. Wang, M. Malac, A. Meldrum, R. Egerton, and J.M. Buriak, in preparation. 
[4] J. Narayan, and B.C. Larson, J. Appl. Phys. 93, 279, (2003).

[5] B.Q. Li, and J.M. Zuo, Phys. Rev. B 72, 85434, (2005).

[6] This research is supported by NINT and NSERC.

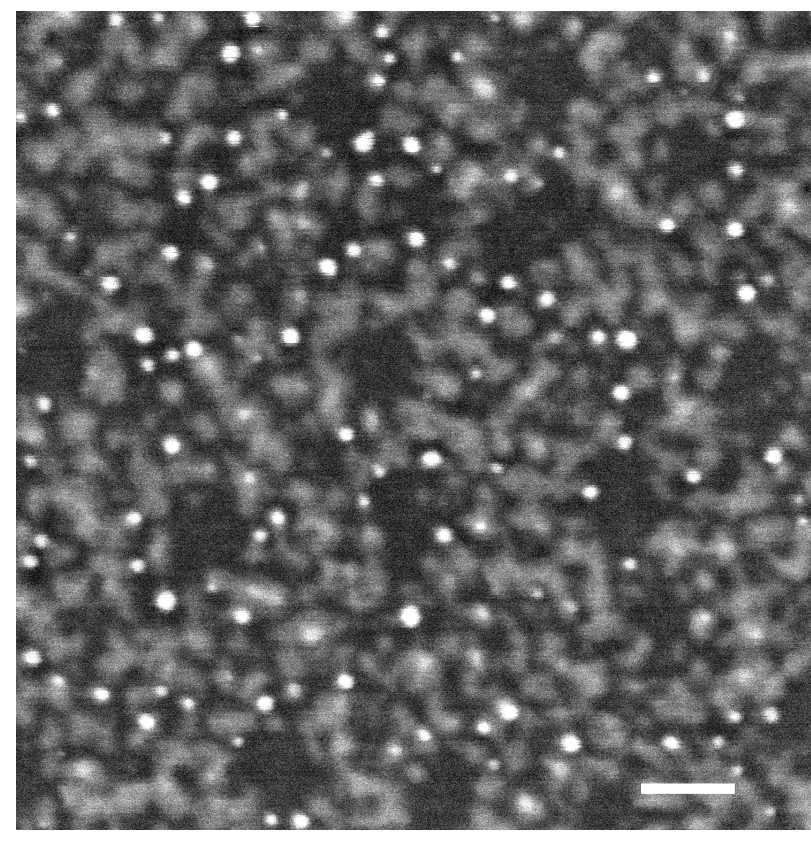

Fig .1. SEM image for Au nanoparticles grown on $\mathrm{Si}(111)$ substrate. (Scale bar: $20 \mathrm{~nm}$ )

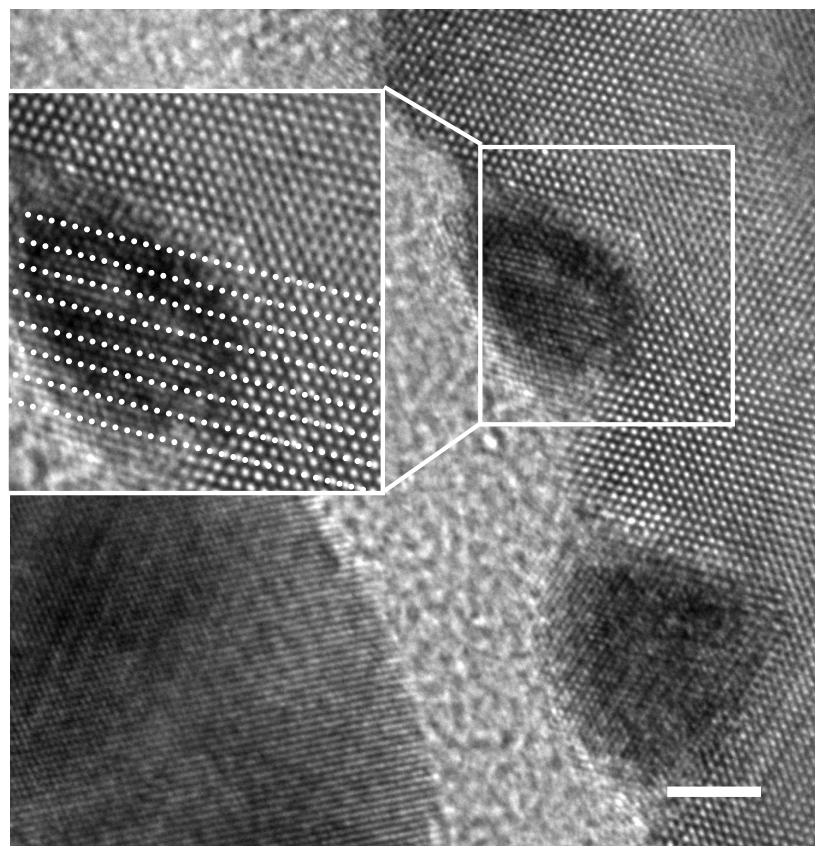

Fig.3. Cross-section TEM image, showing domain match of $\mathrm{Au}(111)$ and $\mathrm{Si}(111)$ planes. (Scale bar: $5 \mathrm{~nm}$ )

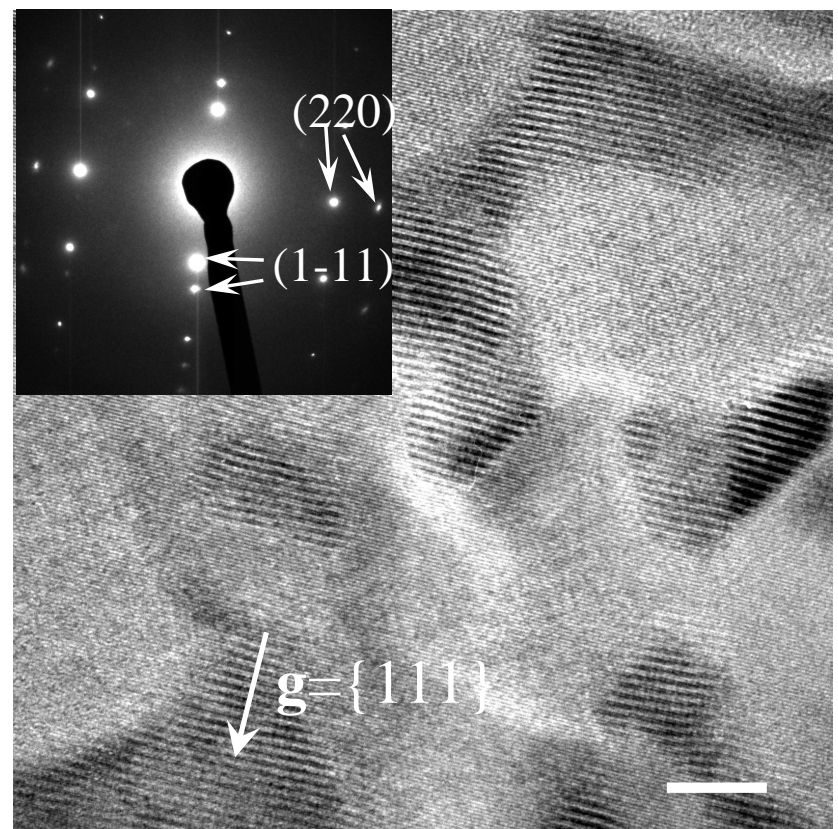

Fig. 2. Moiré fringes and SAED pattern (inset) taken near $\mathrm{B}=[112] .($ Scale bar: $10 \mathrm{~nm})$

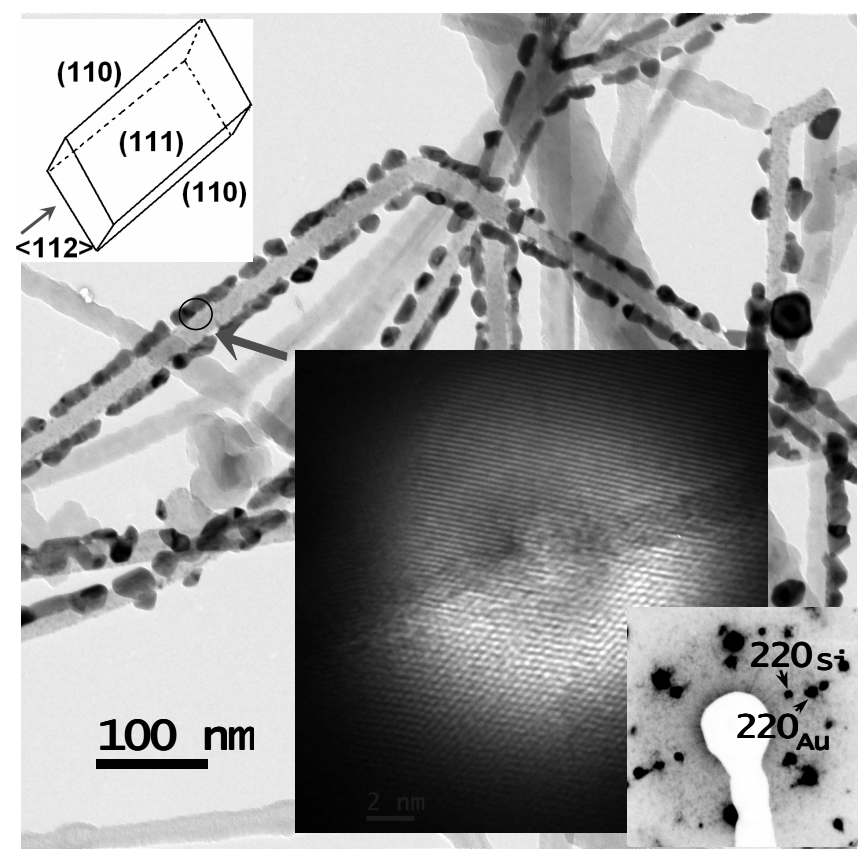

Fig. 4. TEM images and nano-beam diffraction pattern showing the epitaxial and preferential growth of the Au nanoparticles on Si nanowires. 